\title{
An integrated spiral antenna system for UWB
}

\author{
Magnus Karlsson, and Shaofang Gong \\ University of Linköping, Department of Science and Technology - ITN, LiU Norrköping, \\ SE-601 74 Norrköping, Sweden, Phone +46 11363491
}

\begin{abstract}
As wireless communication applications require more and more bandwidth, the demand for wideband antennas increases as well. For instance, the ultra wideband radio (UWB) utilizes the frequency band of 3.110.6 GHz. The spiral antenna has a higher spectral efficiency than other planar antennas like the patch antenna. Theoretically, any type of antennas can be combined into different kind of arrays, in order to improve performance beyond that from one single antenna. The electrically coupled parallelism is one solution to extend bandwidth. By combining two spiral antennas with different radius of the radiation zone, the standing wave ratio (SWR) can be kept low for a large bandwidth, resulting in an improved spiral antenna performance for UWB. Furthermore, a study of how spiral dimensions impact on Gain and SWR was conducted and presented.
\end{abstract}

\section{INTRODUCTION}

The spiral antenna has wide bandwidth, i.e., good spectral efficiency compared to other planar antennas [1]. Spiral antennas are based on Archimedes principle for a spiral, which can have many shapes depending on design goals. Theoretically a spiral antenna with an infinite number of turns with optimal spacing between arms has infinite spectral efficiency and bandwidth. Practically we need to deal with the fact that the unlimited size is not possible, and the turns cannot be too close to each other without suffering the gain. The antenna is intended for a module design in which other components will be placed on the backside of the module. Thus a ground plane is used to shield components from the antenna radiation. Therefore the antennas should be integrated on a multilayer PCB (Printed Circuit Board). Ultra wideband radio $(\mathrm{UWB})$ has been specified in the frequency range 3.1 to $10.6 \mathrm{GHz}$ [2].

\section{OVERVIEW OF THE ANTENNA SYSTEMS}

\section{A. Antenna Solutions}

An antenna array is often used to extend antenna performance when that from a single antenna element is not sufficient. No matter which array technique used, most antennas should be connected to a $50 \Omega$ port. Fig. 1 shows the schematics and layouts of our proposal to utilize one monofilar spiral antenna or two monofilar spiral antennas in parallel for UWB. A power divider is used in the dual antenna array solution.
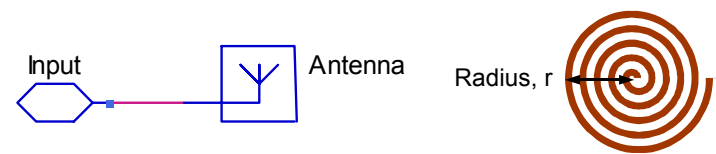

(a) Single antenna solution
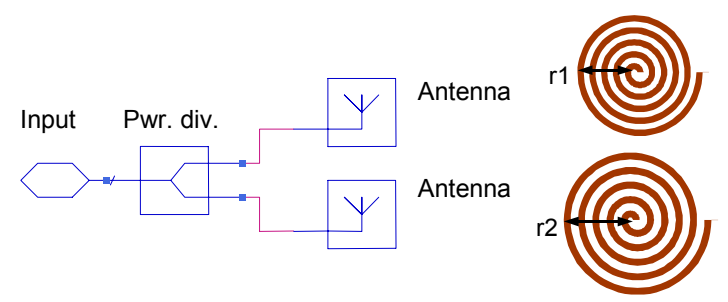

(b) Dual antenna array solution

Fig. 1. Overview of the antenna system: (a) single antenna solution, and (b) array solution.

\section{B. Substrate}

Table 1. Four layer PCB parameters

\begin{tabular}{|l|l|l|}
\hline Dielectric top & RCC & Height, $\mathrm{h}=0.05 \mathrm{~mm}$ \\
\hline Dielectric core & RO4350B & $(1 \mathrm{x}) \mathrm{h}=1.524 \mathrm{~mm}$ \\
\hline Dielectric bottom & RCC & $\mathrm{h}=0.05 \mathrm{~mm}$ \\
\hline Dielectric constant & All layers & $3.48 \pm 0.05$ \\
\hline Dissipation factor & All layers & 0.004 \\
\hline Metal thickness & Top and bottom & $25 \mu \mathrm{m}$ \\
\hline Metal thickness & RO4350B (core) & $18 \mu \mathrm{m}$ \\
\hline Metal conductivity & All layers & $5.8 \times 10^{7} \mathrm{~S} / \mathrm{m}$ \\
\hline Surface roughness & All layers & $0.001 \mathrm{~mm}$ \\
\hline
\end{tabular}

Table 1. shows material parameters used for simulations, and Fig. 2 illustrates the cross section of the PCB. Rogers RO43xx series are used for high frequency PCBs suitable for radio frequency (RF) modules [3].

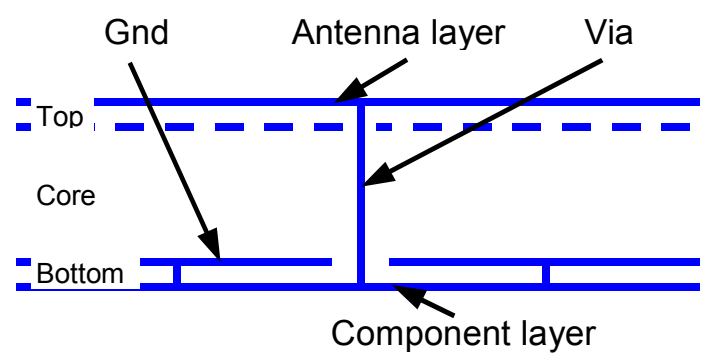

Fig. 2. Cross section of the four layer PCB

\section{Principle of the Monofilar Spiral}

As shown in Fig. 3, the circumference of the radiation zone determines the radiation frequency. The circumference should be $>2 \lambda$ [4]. If the current flowing backwards is smaller than that flowing forward, there is a net current flow through the spiral and radiation occurs.

Feeding to the spiral can be done either from the centre or from the outside. In the designs shown in this paper the feeding is done by a via to the centre of the spiral (see Fig. 2). The input impedance depends on the line width together with the distance to the ground plane, because the characteristic impedance of the spiral arm is dependant of the line width as in the case of a microstrip line. The real part of the input impedance can be controlled by the line width, while the imaginary part is 
more difficult to control. If implemented in a narrow band system the spiral antenna can be matched with a classical RF matching technique for optimal performance in that frequency region. However if a wideband operation is required the issue must be solved with other techniques.

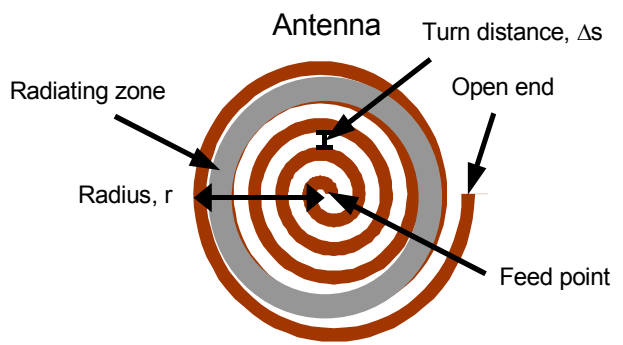

Fig. 3. Layout of a monofilar spiral antenna

\section{SIMULATION RESULTS}

\section{A. Gain and SWR Design Considerations}

Fig. 4 shows gain simulations for various monofilar spiral antennas. Simulations marked with $1 \mathrm{x}$ are done with substrate definitions exactly as displayed in table 1 and Fig. 2. The simulation marked with $2 x$ has the same material but with a double core layer height. Thus, the height from the antenna plane to the ground plane is 1.5 and $3.0 \mathrm{~mm}$, respectively. The substrate marked with $2 \mathrm{x}-$ air is the $1 \mathrm{x}$ substrate with a $1.5 \mathrm{~mm}$ air distance added between the ground plane and the PCB. Fig. 4a shows a series of simulations about how the distance between turns affects the antenna gain. It is seen that if the turn distance decreases the gain of the spiral antenna suffers. Fig. $4 \mathrm{~b}$ shows that a larger number of turns improves the antenna gain, but the density of turns should not increase. Fig. 4c shows how the dielectric loss and the substrate thickness impact on the gain.

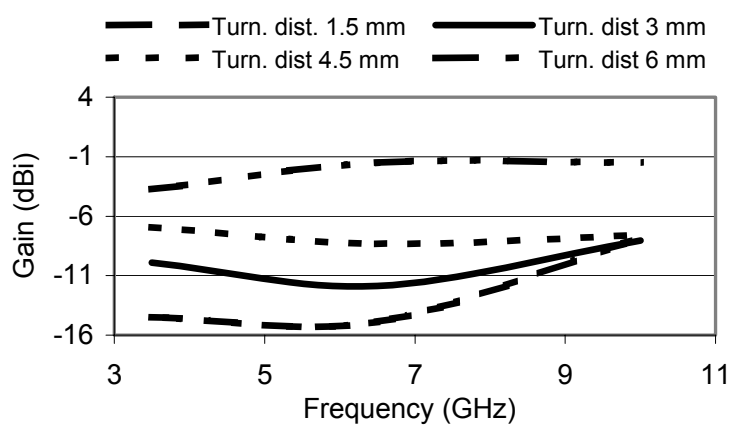

(a) Gain vs. the turn distance, $1.5 \mathrm{~mm}$ substrate

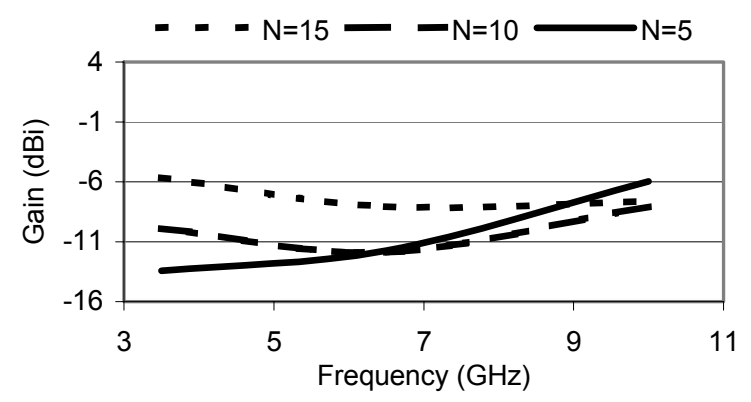

(b) Gain vs. the number of turns

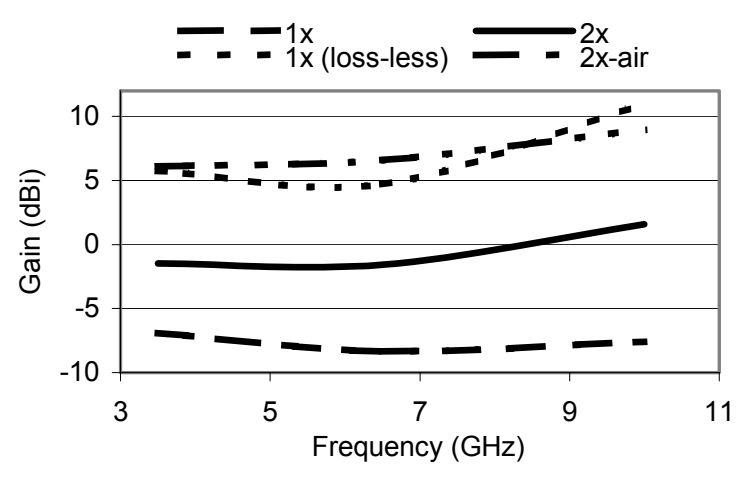

(c) Gain vs. different substrates

Fig. 4. Gain simulations: (a) gain vs. turn distance, (b) gain vs. the number of turns, and (c) gain vs. different substrates.

\section{B. Monofilar Spiral Antenna of $50 \Omega$}

Fig. 5 shows the layout and voltage standing wave ratio (VSWR) simulation of a monofilar spiral antenna. To optimize performance, the real part of the characteristic impedance was calculated to be $50 \Omega$, i.e., the line width was calculated to be $3.43 \mathrm{~mm}$ with a substrate thickness of $1.5 \mathrm{~mm}$. This would be optimal for all frequencies if the input impedance was kept stable for all frequencies. Three radiuses with three different turn distances for each size of the antenna were designed and simulated. It is shown in all three of the simulations in Fig. 5b-d that a more dense turn distance $(\Delta s)$ provides a higher spectral efficiency. As mentioned in the introduction, in theory the infinite number of turns gives the infinite spectral efficiency. However, owing to the chosen line width and the need of spacing between turns and limited number of turns, the bandwidth is limited in our case as seen in Fig. 5b-d. Fig. 5e-h shows radiation patterns at $6.5 \mathrm{GHz}$, one cut from each antenna size and one with air core substrate is shown. The air core antenna shown in Fig. 5h has higher efficiency performance compared to Fig. 5d-g, i.e., gain relative to directivity.

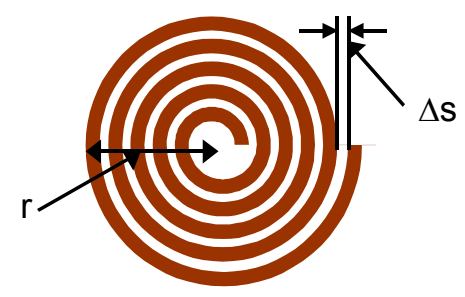

(a) Layout

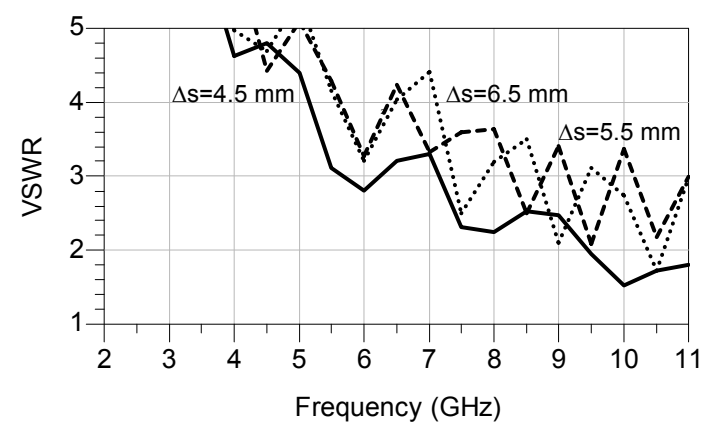

(b) Simulation, $\mathrm{r}=3 \mathrm{~cm}$ 


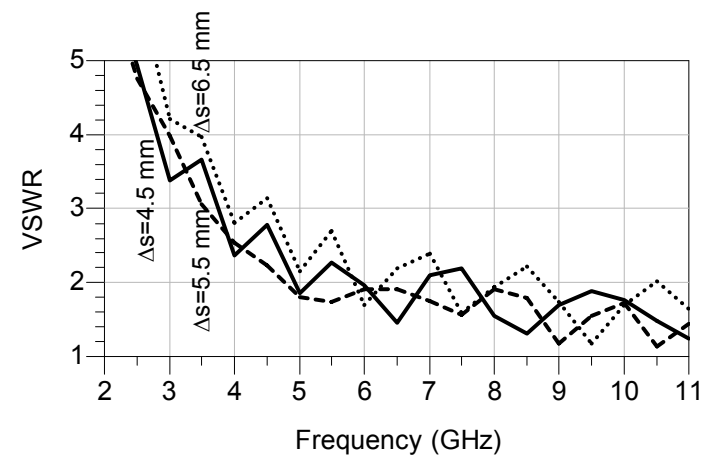

(c) Simulation, $\mathrm{r}=5 \mathrm{~cm}$

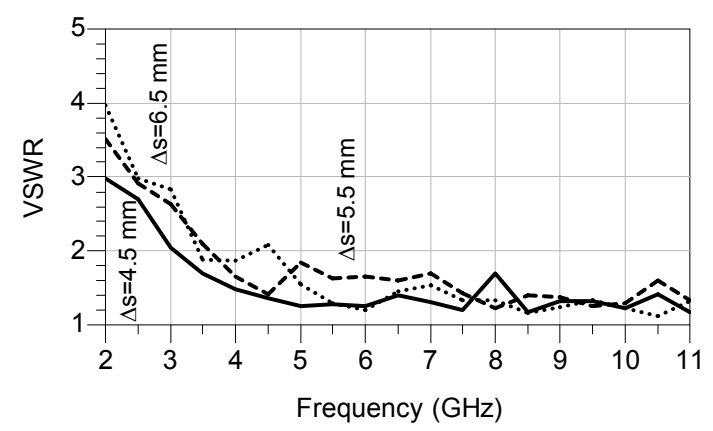

(d) Simulation, $r=7.5 \mathrm{~cm}$

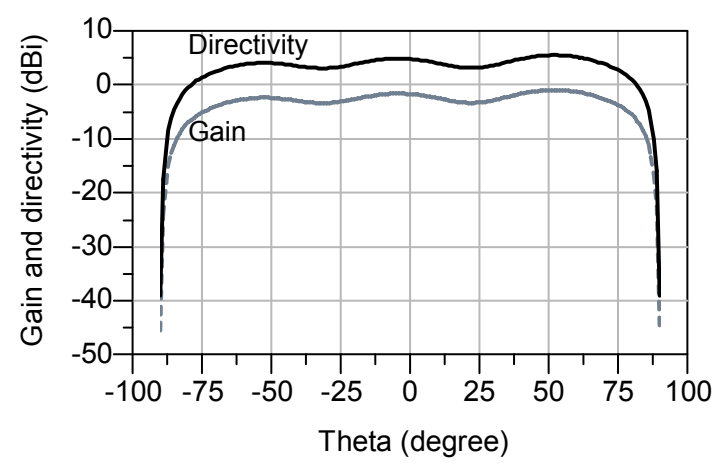

(e) Simulation at $6.5 \mathrm{GHz}$

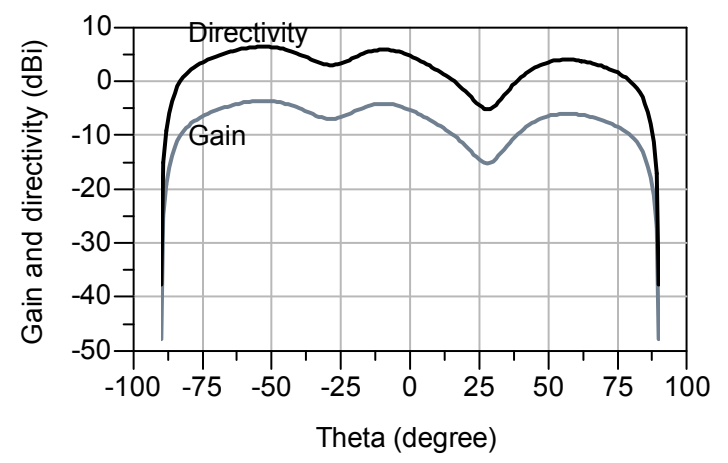

(f) Simulation at $6.5 \mathrm{GHz}$

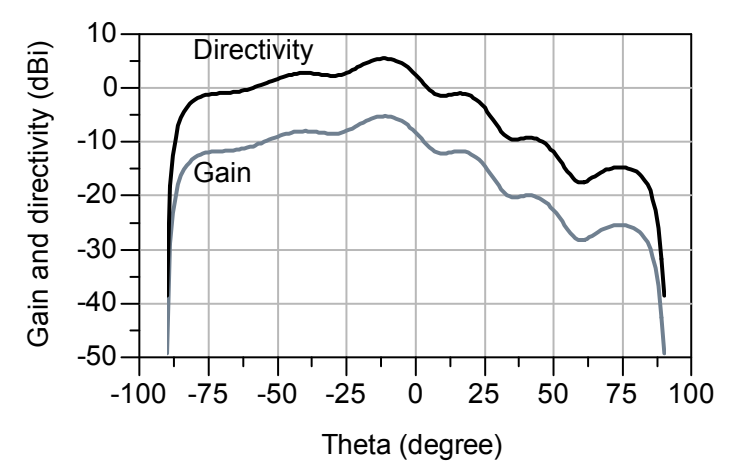

(g) Simulation at $6.5 \mathrm{GHz}$

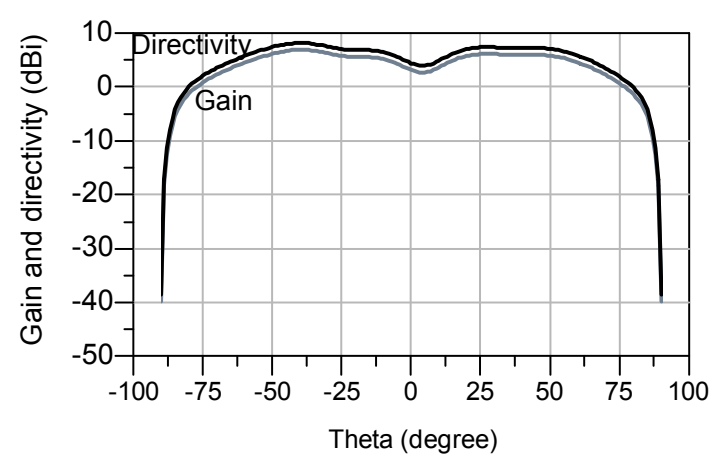

(h) Simulation at $6.5 \mathrm{GHz}$ with an additional $1.5 \mathrm{~mm}$ air core, $\mathrm{r}=7.5 \mathrm{~cm}$

Fig. 5. Monofilar spiral antenna of $50 \Omega$ input impedance: (a) Layout, (b) VSWR simulation, $r=3 \mathrm{~cm}$, (c) VSWR simulation, $r=5 \mathrm{~cm}$, (d) VSWR simulation, $r=7.5 \mathrm{~cm}$, (e) radiation simulation, $\mathrm{r}=3 \mathrm{~cm}, \Delta \mathrm{s}=4.5 \mathrm{~mm}$, (f) radiation simulation, $\mathrm{r}=5 \mathrm{~cm}, \Delta \mathrm{s}=4.5 \mathrm{~mm},(\mathrm{~g})$ radiation simulation, $\mathrm{r}=7.5$ $\mathrm{cm}, \Delta \mathrm{s}=4.5 \mathrm{~mm}$, and $(\mathrm{h})$ radiation simulation with an additional $1.5 \mathrm{~mm}$ air core, $\mathrm{r}=7.5 \mathrm{~cm}$.

\section{Pair of Monofilar Spiral Antenna of $100 \Omega$}

Using a similar approach as in Section III-B, two antennas optimized for the $100 \Omega$ input were designed. To use the antenna in a $50 \Omega$ system either a matching network is utilized or an array configuration shown in Fig. $1 \mathrm{~b}$ is used. The radius of the antennas is 73 and 84 $\mathrm{mm}$, respectively. Fig. 6a shows the layout, and Fig. $6 \mathrm{~b}$ shows the VSWR simulation. Gain figures are seen in Fig. $4 c$, the line marked with $2 x$.

The real part of the combined input impedance is designed close to $50 \Omega$ in a wide frequency band. The VSWR curve of the array is under or between the VSWR curves of the two separate antennas of $100 \Omega$. As seen in Fig. 6c the arrays have a bandwidth between 1.79-11 $\mathrm{GHz}$ and $1.8-11 \mathrm{GHz}$ at $\operatorname{VSWR}<2$, turn dist. $=4.5 \mathrm{~mm}$ and $6 \mathrm{~mm}$, respectively. Fig. $6 \mathrm{~d}$ shows the gain of the antenna array, the substrate is $2 \mathrm{x}$ and $2 \mathrm{x}$-air, respectively.

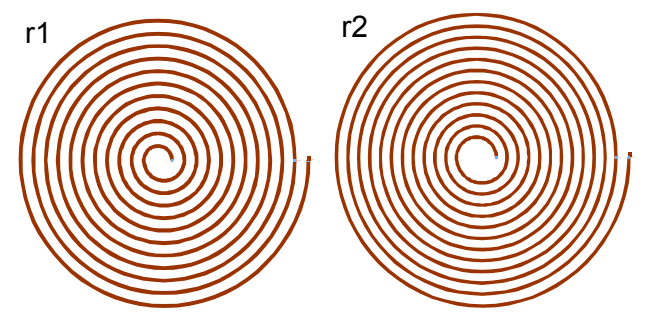

(a) Layout, r1=73 $\mathrm{mm}$ and $\mathrm{r} 2=84 \mathrm{~mm}$ 


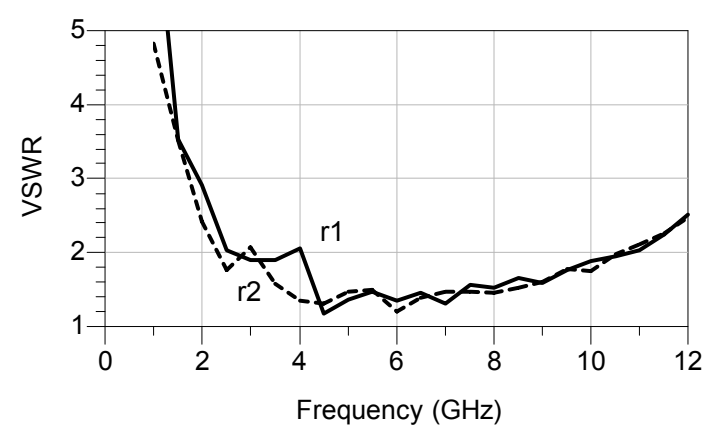

(b) Simulation, separate antennas

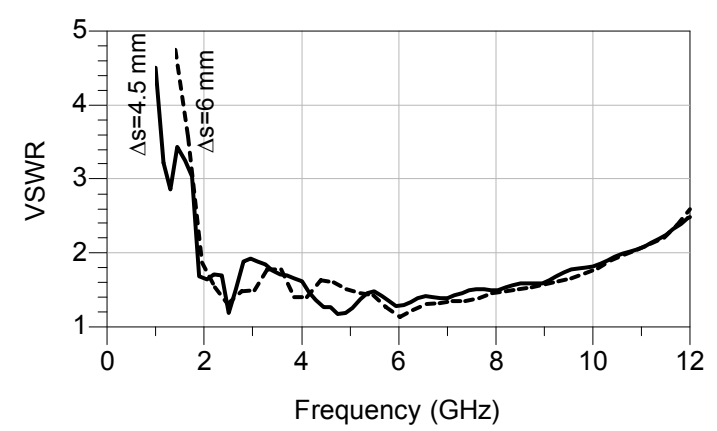

(c) Simulation, of two combined antennas

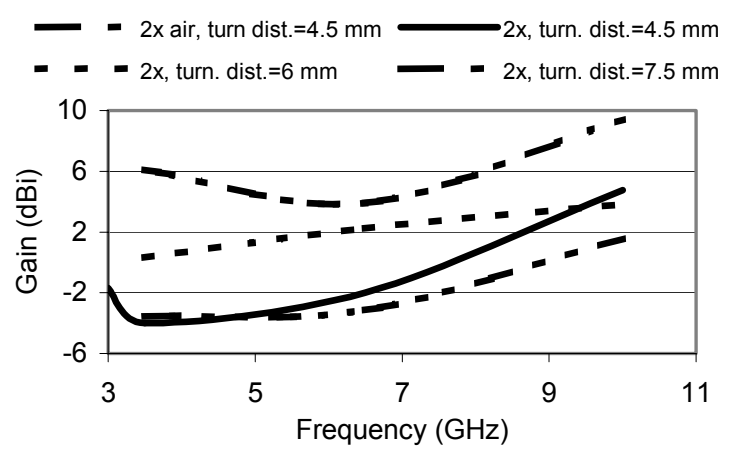

(d) Gain simulation, $2 x$ and $2 x$ air substrate

Fig. 6. $\quad 100 \Omega$ spiral antennas: (a) layout of the two spiral antennas, (b) simulation of the spirals with radius $\mathrm{r} 1$ and $\mathrm{r} 2$, (c) VSWR simulation of the combined inputs, and (d) simulation of gain of the dual antenna arrays.

\section{DISCUSSION}

A single monofilar spiral antenna for UWB is presented in Section III-B, see Fig. 5. The antenna covers the entire UWB frequency band of 3.1-10.6 GHz at various SWR qualities, i.e., the physical size limits the spectral efficiency and determines the centre frequency.

To achieve a wider bandwidth than that from the single spiral, a pair of monofilar spiral antennas is presented in Section III-C. It is shown in Fig. 6c that the two combined spirals cover the entire UWB frequency range with a $\operatorname{VSWR}<2$.

In the gain simulation shown in Fig. 6d it is seen that the gain of the $2 \mathrm{x}$ array (turn dist. $=4.5 \mathrm{~mm}$ ) is slightly lower than that from the single antenna for frequencies lower than $7 \mathrm{GHz}$, but higher for frequencies above 7 GHz. This is probably due to a better coherent interference between the two antennas in the upper frequency range. It is also seen in Fig. 6d that the antenna pair with the $2 \mathrm{x}$ air substrate gives higher gain than that with the $2 x$ substrate. Furthermore it is shown that turn distance is important for gain performance, see Fig $6 \mathrm{~d}$ and Fig 4.

\section{CONCLUSIONS}

- A study of monofilar spiral antenna for UWB has been conducted. How the substrate loss and thickness affect the antenna gain were shown. Gain improvement with an air gap between the ground plane and the antenna plane was shown, see Fig 4.

- Monofilar spiral antennas for UWB, optimized for an input impedance of $50 \Omega$ was designed and simulated. It is shown that a planar monofilar spiral antenna implemented on a PCB is suitable for RF module designs.

- Monofilar spiral antennas with a radius of $3 \mathrm{~cm}$ were designed and simulated. It is shown that one monofilar spiral antenna of this size covers partially the UWB frequency band, i.e., above $5.1 \mathrm{GHz}$ at $\operatorname{VSWR}<3$.

- Monofilar spiral antennas with a radius of $5 \mathrm{~cm}$ were designed and simulated. It is shown that one monofilar spiral antenna of this size is sufficient to cover the entire UWB frequency band, i.e., 3.1-10.6 GHz at VSWR $<3.4$. The majority of the frequency band has a VSWR $<3$, see Fig. 5c.

- Monofilar spiral antennas with a radius of $7.5 \mathrm{~cm}$ were designed and simulated. It is shown that one monofilar spiral antenna of this size is sufficient to cover the hole UWB frequency band, i.e., 3.1-10.6 GHz at VSWR $<2$.

- Dual antenna arrays of monofilar spiral antennas for UWB with an input impedance of $50 \Omega$ was simulated. It is shown that a pair of monofilar spirals can be used in parallel to extend the bandwidth, i.e., $1.79-11 \mathrm{GHz}$ at VSWR $<2$, see Fig. 6c.

\section{REFERENCES}

[1] E. Gschwendtner, D. Löffler, and W. Wiesbeck, "Spiral antenna with external feeding for planar applications", Africon, 1999 IEEE, vol. 2, 28 Sept.-1 Oct. pp. 1011-1014, 1999.

[2] G. R. Aiello and G. D. Rogerson, "Ultra Wideband Wireless Systems”, IEEE microwave magazine, pp. 36-47, June 2003

[3] M. Karlsson and S. Gong, "Wideband patch antenna array for multi-band UWB", Proc. of IEEE 11th SCVT 2004, November 2004.

[4] H. Nakano, Y. Okabe, H. Mimaki, and J. Yamauchi, "A Monofilar Spiral Antenna Excited Through a Helical Wire", IEEE transactions on antennas and propagation, vol. 51, no. 3, March 2003. 